\title{
ANÁLISIS PANBIOGEOGRÁFICO DE VEXILLATA (NEMATODA: ORNITHOSTRONGYLIDAE) Y SUS HUÉSPEDES (MAMMALIA: RODENTIA)
}

\author{
Tania Escalante, ${ }^{1 *}$ Elizabeth A. Martínez-Salazar, ${ }^{2}$ Jorge Falcón-Ordaz, ${ }^{3}$ \\ Miguel LINAJE ${ }^{4} \&$ Ricardo GUERRERO ${ }^{5}$ \\ ${ }^{1}$ Museo de Zoología “Alfonso L. Herrera”, Departamento de Biología Evolutiva, Facultad de Ciencias, \\ Universidad Nacional Autónoma de México, Apdo. Postal 70-399, 04510 México, D. F., México. \\ *tee@ibiologia.unam.mx \\ ${ }^{2}$ Department of Integrative Biology, University of Guelph, Guelph, Ontario, N1G 2W1, Canada. \\ eamsmex@hotmail.com \\ ${ }^{3}$ Universidad Autónoma del Estado de Hidalgo, Centro de Investigaciones Biológicas, Apdo. Postal \\ 1-69, 42001 Pachuca, Hidalgo, México. jfalcon.ordaz@gmail.com \\ ${ }^{4}$ Laboratorio de Sistemas de Información Geográfica, Departamento de Zoología, Instituto de Biología, \\ Universidad Nacional Autónoma de México. Apdo. Postal 70-153, 04510 México, D. F., México. \\ mlinaje@yahoo.com.mx \\ ${ }^{5}$ Instituto de Zoología y Ecología Tropical, Facultad de Ciencias, Universidad Central de Venezuela, \\ Apdo. Postal 47058, Caracas 1041. parasite@cantv.net \\ *Autor de correspondencia: tee@ibiologia.unam.mx
}

Escalante, T., E. A. Martínez-Salazar, J. Falcón-Ordaz, M. Linaje \& R. Guerrero. 2011. Análisis panbiogeográfico de Vexillata (Nematoda: Ornithostrongylidae) y sus huéspedes (Mammalia: Rodentia). Acta Zool. Mex. (n. s.), 27(1): 25-46.

RESUMEN. Se llevó a cabo un análisis panbiogeográfico, a partir de un análisis de parsimonia de endemismos (PAE) de las especies de helmintos parásitos intestinales del género Vexillata (Nematoda: Ornithostrongylidae) y sus especies de huéspedes, mamíferos de las familias Geomyidae y Heteromyidae. Se realizaron dos análisis, el primero empleando solo aquellas especies del género Vexillata con más de dos localidades, y el segundo, analizando todas las localidades de este género de parásito en un solo trazo individual. Se obtuvieron tres trazos generalizados a partir del PAE del primer análisis: costa norte de Venezuela, América Central y Neártico. Solo uno de los trazos fue sustentado por una especie de parásito y su huésped ( $V$. tejerai y Heteromys anomalus). Se propone la existencia de un nodo biogeográfico en Colombia, entre el trazo de la costa norte de Venezuela y el de América Central en el noroeste de Colombia, en los límites con Panamá; y otro en la zona central de México. Estos trazos y nodos coinciden con algunos propuestos por otros autores. En general se observa que estos sistemas pueden ser explicados como tres componentes bióticos. En particular el de América del Sur puede corresponder con aquellas que especiaron por aislamiento (V. scorzai), y especies con distribuciones amplias (V. tejerai), quizá más relacionadas a etapas de movilidad de sus huéspedes (Heteromys).

Palabras clave: helmintos parásitos, nemátodos, mamíferos, panbiogeografía, provincias biogeográficas.

Recibido: 27/10/2009; aceptado: 01/09/2010. 
Escalante, T., E. A. Martínez-Salazar, J. Falcón-Ordaz, M. Linaje \& R. Guerrero. 2011. Panbiogeographic analysis of Vexillata (Nematoda: Ornithostrongylidae) and its hosts (Mammalia: Rodentia). Acta Zool. Mex. (n. s.), 27(1): 25-46.

ABSTRACT. A panbiogeographic analysis was carried out, based on a parsimony analysis of endemicity (PAE) to analyze the species of helminth intestinal parasites of the genus Vexillata (Nematoda: Ornithostrongylidae) and their host species, mammals of the families Geomyidae and Heteromyidae. Two analyses were undertaken, the first using only those species of Vexillata with more than two localities, and the second analyzing all localities of this genus as a single track. Three generalized tracks were obtained by the first PAE: northern coast of Venezuela, Central America and Nearctic. Only one track was identified by a parasite and its host ( $V$. tejerai and Heteromys anomalus). It proposes the existence of two biogeographical nodes, the first is located between the northern coast of Venezuela and Central America, on the boundaries of northeast Colombia and Panama (Colombia node); and the other in Central Mexico. Those track and nodes overlap with some proposed by other authors. In general, it appears that these systems can be explained as three biotic components. In the case of the South American component might correspond with the isolation of species ( V. scorzai), as well as by species that had expanded their geographical distribution ( $V$. tejerai), it could be related to mobilism stages of their host (Heteromys).

Key words: helminths parasites, nematodes, mammals, panbiogeography, biogeographical provinces.

\section{INTRODUCCIÓN}

El género Vexillata (Nematoda: Ornithostrongylidae) está constituido por 14 especies (Durette-Desset \& Digiani 2005, Falcón-Ordaz \& García-Prieto 2005, Falcón-Ordaz et al. 2006), las cuales son parásitos intestinales de mamíferos de las familias Geomyidae (Thomomys, Geomys y Cratogeomys), Heteromyidae (Perognathus, Chaetodipus y Heteromys) y Leporidae (Sylvilagus) (ver Cuadro 1). Las dos primeras se distribuyen desde el sur de Canadá (British Columbia, Alberta, Manitoba y Saskatchewan) hasta el sur de Panamá (Geomyidae) y norte de Venezuela, Colombia y la costa pacífica de Ecuador (Heteromyidae). La familia Leporidae se distribuye, en el Nuevo Mundo, al sur de Canadá pasando por los Estados Unidos de América, México y América Central hasta el sur de Brasil y el norte de Argentina (Anderson et al. 2002, Hall 1981, Schmidly et al. 1993, Villa \& Cervantes 2003, Wilson \& Reeder 2005). Respecto a la distribución de las especies de Vexillata solo se conocen algunas localidades donde han sido recolectadas, y no existen trabajos analizando sus áreas de distribución (Durette-Desset \& Digiani 2005, Falcón-Ordaz \& García-Prieto 2005).

Los parásitos son valiosos indicadores históricos y ecológicos (Hoberg 1997, Brooks et al. 2001), cuyo estudio permite comprender las interacciones multidimensionales entre los factores ecológicos y patrones de distribución, e inclusive ayudan a dilucidar la historia compleja de regiones y biotas (Brooks \& McLennan 1993, Brooks et al. 2001). En particular, los estudios sobre biogeografía histórica en parásitos parten de la premisa que éstos son indicadores históricos y ecológicos sobre la relación huésped-parásito, debido a que su distribución geográfica se limita a aquellas regiones en las cual sus huéspedes obligatorios se co-distribuyen para completar sus 
ciclos de vida (Brooks \& MacLennan 1993), por lo cual el estudio de estos sistemas representa un importante desafío dentro de la biogeografía histórica.

Pocos son los trabajos que han abordado estudios biogeográficos con helmintos parásitos en México, los cuales se han enfocado principalmente a la identificación de patrones de distribución (ej. Vidal-Martínez \& Kennedy 2000, Pérez-Ponce de León 2003, Pérez-Ponce de León \& Choudhury 2002, 2005, Rosas-Valdez et al. 2007), riqueza (ej. Aguilar-Aguilar et al. 2008) y endemismo (ej. Aguilar-Aguilar et al. 2003). En particular, existen tres trabajos con análisis panbiogeográficos que se han enfocado principalmente al sistema parásito-peces dulceacuícolas en México (Rosas-Valdez \& Pérez-Ponce de León 2005, 2008, Martínez-Aquino et al. 2007; ver más adelante). Finalmente, existe otro sistema de parásitos no helmintos, que ha sido analizado por Morrone \& Gutiérrez (2005), con ectoparásitos hematófagos de mamíferos a partir de datos de distribución. Estos autores analizaron los patrones de diversificación de pulgas que habitan la Zona de Transición Mexicana, los cuales coinciden con los de sus huéspedes.

La panbiogeografía (Croizat 1958, 1964) es un enfoque de la biogeografía que enfatiza la importancia de la dimensión geográfica de la biodiversidad para el entendimiento apropiado de la evolución (Morrone \& Gutiérrez 2005). Se ha sugerido que éste enfoque puede emplearse para una exploración inicial de los datos, antes de llevar a cabo un análisis biogeográfico cladístico (Morrone 2004). Rosas-Valdez \& Pérez-Ponce de León (2005) emplearon este método para explicar los patrones biogeográficos de los parásitos de ictalúridos en América del Norte, encontrando que la historia biogeográfica de los helmintos es compleja en la cuenca hidrográfica del río Mississippi, donde se concentra la mayor diversidad de especies de helmintos y la mayor diversidad de ictalúridos, con base en los nodos encontrados. Así mismo los autores sugieren que en México, los helmintos analizados en ese trabajo se encuentran integrados en un componente biótico neártico.

Martínez-Aquino et al. (2007) centraron sus análisis panbiogeográficos en organismos dulceacuícolas (peces-helmintos-plantas acuáticas) distribuidos en la Faja Volcánica Transmexicana, para detectar áreas para conservación. Ellos identificaron cuatro nodos, de los cuales solo el manantial de la Mintzita y el lago de Zacapu presentaron una alta riqueza específica, así como de endemismos, en comparación con el Lago de Opopeo y el manantial de San Cristóbal.

Por otro lado, Rosas-Valdez \& Pérez-Ponce de León (2008) presentan un nuevo estudio sobre los parásitos de Siluriformes, analizando los patrones biogeográficos y de asociación huésped-parásito de los helmintos de grupos neárticos (ictalúridos) y neotropicales (heptaptéridos). Encontraron tres trazos generalizados (norte de México, regiones del río Bravo y sureste de México), que se entrecruzan en dos nodos uno neártico (comprende a la provincia del complejo Tamesí-Pánuco), y otro neárticoneotropical (límites entre el Papaloapan-Coatzacoalcos y Usumacinta de la provincia 
Usumacinta). En este segundo nodo se sitúan los límites de distribución más sureños y norteños de las faunas neárticas y neotropicales de parásitos (ver también Hugot et al. 1994, Guerrero \& Hugot 2003).

El objetivo de este trabajo es analizar mediante panbiogeografía los datos de distribución del género Vexillata y sus huéspedes, para contribuir al conocimiento de sus patrones biogeográficos históricos y analizar si muestran congruencia distribucional.

\section{MATERIAL Y MÉTODOS}

Se construyeron dos bases de datos: una con las localidades de los parásitos y otra para los huéspedes (datos no presentados). En el caso del género Vexillata, se excluyó del análisis a Vexillata noviberae (Dikmans, 1935) Durette-Desset \& Digiani, 2005, por no contar con los datos geográficos de la localidad de recolecta del huésped correspondientes a Leporidae. La base de datos de Vexillata proviene de registros de colecciones (Colección Nacional de Helmintos del Instituto de Biología, UNAM e Instituto de Zoología y Ecología Tropical Universidad Central de Venezuela) y bibliografía (Hall 1916, Caballero \& Cerecero 1943, Caballero 1958, Yoyotte 1972, Durette-Desset 1970, 1978, Denke 1977, Guerrero 1984, Gardner et al. 1994, FalcónOrdaz \& Sanabria-Espinosa 1997, Falcón-Ordaz et al. 2001, 2006, Durette-Dessett \& Digiani 2005, Falcón-Ordaz \& García-Prieto 2005) (Cuadro 1 y Apéndice).

Los datos de distribución de los mamíferos fueron integrados a partir de diferentes fuentes (Cuadro 1 y Apéndice): una base de datos (Mammex; Escalante et al. inédita) compilada en el Laboratorio de Sistemas de Información Geográfica del Instituto de Biología de la UNAM, para el caso de las especies de distribución exclusiva de México; y las bases de datos de Manis (Mammal Networked Information System, http://manisnet.org/) y GBIF (Global Biodiversity Information Facility, http://www. gbif.org/) para las especies con distribución más amplia que México (para la lista de las colecciones consultadas ver Agradecimientos).

Los datos geográficos fueron depurados y mapeados con el programa ArcView 3.2 (ESRI 1999). Dado que algunas de las especies de Vexillata no son susceptibles para ser analizadas mediante panbiogeografía, puesto que poseen una o dos localidades, se realizaron dos análisis: (1) considerando solo aquellas especies con más de dos localidades con las que se elaboraron sus trazos individuales (se excluyeron en este caso a $V$. brooksi, $V$. chabaudi, $V$. chitwoodi, $V$. geomyos, $V$. noviberae, $V$. scorzai y Vexillata sp.; ver número de localidades analizadas en el Apéndice I; Vexillata sp. se excluyó debido a que la taxonomía ha sido controversial); y (2) analizando todas las localidades del género en un solo trazo individual. Para los huéspedes, a partir de las localidades de cada especie se dibujaron los trazos individuales. Todos los trazos individuales fueron obtenidos usando la herramienta Trazos2004 (Rojas-Parra 2007). 
Cuadro 1. Especies del género Vexillata, sus huéspedes y localidades de recolecta.

\begin{tabular}{|c|c|c|}
\hline Parásito & Huésped & Localidad \\
\hline $\begin{array}{l}\text { V. armandae Gardner, } \\
\text { Fong, Banna \& } \\
\text { Raymond, } 1994\end{array}$ & $\begin{array}{l}\text { Chaetodipus hispidus } \\
\text { (Baird,1858) }\end{array}$ & $\begin{array}{l}4.7 \text { mi. S de Portales, Roosvelt Co., Nuevo } \\
\left.\text { Mexico, EUA ( } 34^{\circ} 10^{\prime} 00^{\prime}{ }^{\prime} \mathrm{N}, 103^{\circ} 21^{\prime} 00^{\prime} \mathrm{O}\right) \text {. }\end{array}$ \\
\hline \multirow[t]{2}{*}{$V$. cf. armandae* } & Ch. hispidus & $\begin{array}{l}\text { Waco Co., Texas. EUA }\left(31^{\circ} 32^{\prime} 57^{\prime} ’ \mathrm{~N},\right. \\
\left.97^{\circ} 08^{\prime} 47^{\prime} \mathrm{O}\right) \text {. }\end{array}$ \\
\hline & $\begin{array}{l}\text { Perognathus flavus Baird, } \\
1855\end{array}$ & $\begin{array}{l}\text { Atla, Axapusco, México }\left(14^{\circ} 44^{\prime} 57^{\prime} ’ \mathrm{~N},\right. \\
\left.98^{\circ} 41^{\prime} 02^{\prime}, \mathrm{O}\right) \dagger\end{array}$ \\
\hline $\begin{array}{l}\text { V. brooksi Falcón- } \\
\text { Ordaz \& García- } \\
\text { Prieto, } 2005\end{array}$ & $\begin{array}{l}\text { Heteromys } \\
\text { desmarestianus Gray, } \\
1868\end{array}$ & $\begin{array}{l}\text { Guanacaste, Costa Rica }\left(10^{\circ} 57^{\prime} 40^{\prime} ’ \mathrm{~N},\right. \\
\left.85^{\circ} 29^{\prime} 30^{\prime} \mathrm{O}\right) .\end{array}$ \\
\hline \multirow{5}{*}{$\begin{array}{l}\text { V. convoluta* } \\
\text { (Caballero \& } \\
\text { Cerecero, 1943) }\end{array}$} & $\begin{array}{l}\text { Cratogeomys fulvescens } \\
\text { Merriam, } 1895\end{array}$ & $\begin{array}{l}\text { Huamantla, Tlaxcala, México }\left(19^{\circ} 19^{\prime} 09^{\prime \prime} \mathrm{N},\right. \\
\left.97^{\circ} 54^{\prime} 53^{\prime \prime} \mathrm{O}\right) \dagger \text {. }\end{array}$ \\
\hline & $\begin{array}{l}\text { C. merriami (Thomas, } \\
\text { 1893) }\end{array}$ & 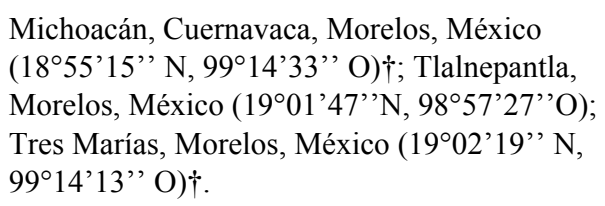 \\
\hline & $\begin{array}{l}\text { C. thylorinus } \\
\text { (Merriam,1895) }\end{array}$ & $\begin{array}{l}\text { Ecatepec, Estado de México, México } \\
\left(19^{\circ} 36^{\prime} 03^{\prime}{ }^{\prime} \mathrm{N}, 99^{\circ} 03^{\prime} 09^{\prime} ’ \mathrm{O}\right)\end{array}$ \\
\hline & Cratogeomys sp. & $\begin{array}{l}\text { Cerro Gordo, Otumba, Estado de México, } \\
\text { México }\left(19^{\circ} 45^{\prime} 07^{\prime}{ }^{\prime} \mathrm{N}, 98^{\circ} 47^{\prime} 58^{\prime \prime}\right) \dagger \text {. }\end{array}$ \\
\hline & $\begin{array}{l}\text { Thomomys umbrinus } \\
\text { (Richardson, 1829) }\end{array}$ & $\begin{array}{l}\text { La Malinche, Tlaxcala, México }\left(19^{\circ} 14^{\prime} 28^{\prime \prime} \mathrm{N} \text {, }\right. \\
\left.97^{\circ} 58^{\prime} 37^{\prime \prime} \mathrm{O}\right) \dagger \text {. }\end{array}$ \\
\hline $\begin{array}{l}\text { V. chabaudi Yoyotte, } \\
1972\end{array}$ & $\begin{array}{l}\text { H. australis Thomas, } \\
1901\end{array}$ & $\begin{array}{l}\text { Pichinde, Valle del Cauca, Colombia } \\
\left(03^{\circ} 26^{\prime} 00^{\prime \prime} \mathrm{N}, 76^{\circ} 37^{\prime} 00^{\prime} \mathrm{O}\right)\end{array}$ \\
\hline $\begin{array}{l}\text { V. chitwoodi Durette- } \\
\text { Desset \& Digiani, } \\
2005\end{array}$ & $\begin{array}{l}\text { Geomys pinetus } \\
\text { (Rafinesque, 1817) }\end{array}$ & $\begin{array}{l}\text { Tallahassee, Florida, EUA }\left(30^{\circ} 26^{\prime} 17^{\prime} ’ \mathrm{~N} \text {, }\right. \\
\left.84^{\circ} 16^{\prime} 51^{\prime \prime} \mathrm{O}\right) \text {. }\end{array}$ \\
\hline $\begin{array}{l}\text { V. dessetae* Denke, } \\
1977\end{array}$ & H. desmarestianus & $\begin{array}{l}\text { Los Tuxtlas, Veracruz, México }\left(18^{\circ} 35^{\prime} 00^{\prime} ’ \mathrm{~N} \text {, }\right. \\
\left.95^{\circ} 07^{\prime} 00^{\prime} \mathrm{O}\right) \text {; Catemaco, Veracruz, Mexico } \\
\left(18^{\circ} 25^{\prime} 49^{\prime}{ }^{\prime} \mathrm{N}, 94^{\circ} 58^{\prime} 09^{\prime}, \mathrm{O}\right) \dagger\end{array}$ \\
\hline $\begin{array}{l}\text { V. geomyos Falcón- } \\
\text { Ordaz, Hsuan-Wien \& } \\
\text { Lamothe-Argumedo, } \\
2006\end{array}$ & $\begin{array}{l}\text { G. attwateri (Tucker y } \\
\text { Schmidly, 1981) }\end{array}$ & $\begin{array}{l}\text { Welder, Wildlife Refuge, San Patricio Co., } \\
\text { Texas, EUA ( } 28^{\circ} 07^{\prime} 15^{\prime}{ }^{\prime}, 97^{\circ} 22^{\prime} 19^{\prime} \text { 'O). }\end{array}$ \\
\hline $\begin{array}{l}\text { V. legalle* Denke, } \\
1977\end{array}$ & H. desmarestianus & $\begin{array}{l}\text { Los Tuxtlas, Veracruz, México }\left(18^{\circ} 35^{\prime} 00^{\prime \prime} \mathrm{N} \text {, }\right. \\
\left.95^{\circ} 07^{\prime} 00^{\prime \prime} \mathrm{O}\right) \text {; Catemaco, Veracruz, México } \\
\left(18^{\circ} 25^{\prime} 49^{\prime}, \mathrm{N}, 94^{\circ} 58^{\prime} 09^{\prime}, \mathrm{O}\right) \dagger .\end{array}$ \\
\hline
\end{tabular}


Cuadro 1. Especies del género Vexillata, sus huéspedes y localidades de recolecta (Continúa).

\begin{tabular}{|c|c|c|}
\hline Parásito & Huésped & Localidad \\
\hline \multirow[t]{2}{*}{$\begin{array}{l}\text { V. liomyos* Falcón- } \\
\text { Ordaz, Gardner \& } \\
\text { Pérez-Ponce de León, } \\
2001\end{array}$} & H. irroratus (Gray, 1868) & $\begin{array}{l}\text { 2km E San Andres Cuajimalpa, Hueyotlipan, } \\
\text { Tlaxcala, México (19²3'17'’N, } \\
\left.98^{\circ} 20^{\prime} 12^{\prime \prime} \mathrm{O}\right) \dagger \text {; Panotla, Tlaxcala, México } \\
\left(19^{\circ} 21^{\prime} 37^{\prime}, \mathrm{N}, 98^{\circ} 15^{\prime} 28^{\prime}{ }^{\prime} \mathrm{O}\right) \text {. }\end{array}$ \\
\hline & H. pictus (Thomas, 1893) & $\begin{array}{l}\text { Chamela, Jalisco, México }\left(19^{\circ} 30^{\prime} 00^{\prime \prime} \mathrm{N} \text {, }\right. \\
\left.105^{\circ} 03^{\prime} 00^{\prime} \mathrm{O}\right) ; 1.4 \text { Kms. al } \mathrm{N} \text { de } \\
\text { Tacote, Nayarit, México }\left(21^{\circ} 23^{\prime} 00^{\prime}, \mathrm{N},\right. \\
\left.104^{\circ} 55^{\prime} 00^{\prime} \mathrm{O}\right) \dagger .\end{array}$ \\
\hline \multirow[t]{2}{*}{$\begin{array}{l}\text { V. noviberae } \\
\text { (Dikmans, 1935) }\end{array}$} & $\begin{array}{l}\text { Sylvilagus floridanus } \\
\text { (J.A.Allen, 1890) }\end{array}$ & Kansas, Louisisana, EUA. \\
\hline & $\begin{array}{l}\text { S. palustris (Bachman, } \\
\text { 1837) }\end{array}$ & Florida, EUA. \\
\hline $\begin{array}{l}\text { V. scorzai Guerrero, } \\
1984\end{array}$ & $\begin{array}{l}\text { H. anomalus (Thompson, } \\
\text { 1815) }\end{array}$ & $\begin{array}{l}\left.\text { La Azulita, Edo. Mérida ( } 8^{\circ} 44^{\prime} \mathrm{N}, 71^{\circ} 27^{\prime} \mathrm{O}\right) \text {, } \\
\text { Venezuela. }\end{array}$ \\
\hline $\begin{array}{l}\text { V. tejerai Guerrero, } \\
1984\end{array}$ & H. anomalus & 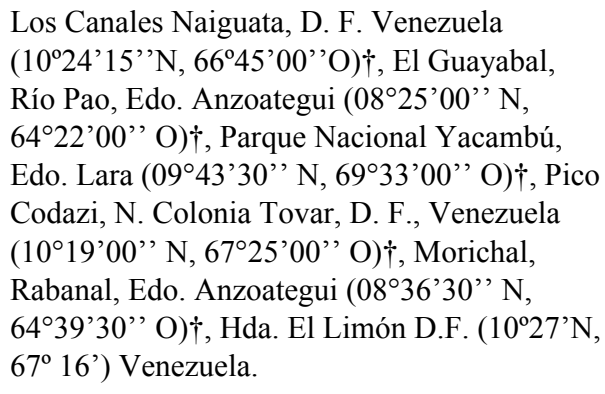 \\
\hline \multirow[t]{5}{*}{$\begin{array}{l}\text { V. vexillata* (Hall, } \\
\text { 1916) }\end{array}$} & H. irroratus (Gray, 1868) & $\begin{array}{l}\text { Huhuetla, Hidalgo, México }\left(20^{\circ} 27^{\prime} 69^{\prime} ’ \mathrm{~N} \text {, }\right. \\
\left.98^{\circ} 04^{\prime} 42^{\prime \prime} \mathrm{O}\right) \text {. }\end{array}$ \\
\hline & H. pictus (Thomas, 1893) & $\begin{array}{l}\text { Mapastepec, Chiapas, México }\left(15^{\circ} 27^{\prime} 00^{\prime \prime} \mathrm{N}\right. \\
\left.92^{\circ} 53^{\prime} 00^{\prime \prime} \mathrm{O}\right) \text {. }\end{array}$ \\
\hline & H. salvini (Thomas, 1893) & $\begin{array}{l}\text { Guanacaste, Costa Rica }\left(10^{\circ} 57^{\prime} 40^{\prime \prime} \mathrm{N},\right. \\
\left.85^{\circ} 29^{\prime} 30^{\prime} \mathrm{O}\right) \dagger\end{array}$ \\
\hline & $\begin{array}{l}\text { Th. bottae (Eydoux } \\
\text { Gervais, 1836) }\end{array}$ & $\begin{array}{l}\text { Albuquerque, Bernardillo Co., Nuevo Mexico, } \\
\text { EUA. }\left(35^{\circ} 011^{\prime} 11^{\prime \prime} \mathrm{N}, 106^{\circ} 43^{\prime} 13^{\prime \prime} \mathrm{O}\right) \dagger \text {. }\end{array}$ \\
\hline & $\begin{array}{l}\text { Th. talpoides Richardson, } \\
1829\end{array}$ & $\begin{array}{l}\text { Livermore Co., Colorado, EUA. ( } 40^{\circ} 47^{\prime} 40^{\prime}{ }^{\circ} \mathrm{N} \text {, } \\
105^{\circ} 13^{\prime} 00^{\prime} \text { 'O); Hill Co., Nuevo Mexico, EUA } \\
\left(32^{\circ} 50^{\prime} 04^{\prime \prime} \mathrm{N}, 108^{\circ} 04^{\prime} 42^{\prime \prime} \mathrm{O}\right) \text {. }\end{array}$ \\
\hline Vexillata sp. & H. desmarestianus & $\begin{array}{l}\text { Guanacaste, Costa Rica. }\left(10^{\circ} 57^{\prime} 40^{\prime \prime} \mathrm{N},\right. \\
\left.85^{\circ} 29^{\prime} 30^{\prime \prime} \mathrm{O}\right) \text {; Finca El Helechales, Puntarenas, } \\
\text { Costa Rica. }\left(09^{\circ} 04^{\prime} 34^{\prime}{ }^{\prime} \mathrm{N}, 83^{\circ} 05^{\prime} 31^{\prime \prime} \mathrm{O}\right) \dagger .\end{array}$ \\
\hline
\end{tabular}

*Especies registradas en México, $\dagger$ Nuevas localidades registradas en este trabajo. 
Para obtener los trazos generalizados, se llevó a cabo un Análisis de Parsimonia de Endemismos (PAE; Morrone 1994, Luna-Vega et al. 2000). El PAE es un método de la biogeografía histórica que permite agrupar áreas, con base en una analogía con la sistemática filogenética, de acuerdo con sus taxones compartidos, al emplear un algoritmo de parsimonia (Crisci et al. 2000). En un PAE panbiogeográfico, los taxones están representados por los trazos individuales. Así, en un cladograma obtenido de un PAE aplicado en panbiogeografía, dos o más trazos individuales representarán un trazo generalizado si se encuentran como sinapomorfías para un mismo clado.

Para el PAE, cada trazo individual fue superpuesto sobre el mapa de las provincias biogeográficas de Morrone (2001, 2006), con lo que se construyó una matriz de datos de presencias " 1 " y ausencias " 0 ", donde las columnas corresponden a las especies y las filas a las provincias que atraviesa el trazo individual de cada especie (la matriz de datos puede solicitarse directamente a los autores). En los dos análisis se emplearon 25 provincias bióticas y 16 especies de mamíferos; en el primer PAE se usaron los trazos de siete especies de Vexillata $(V$. armandae, $V$. convoluta, $V$. dessetae, $V$. legalle, $V$. liomyos, $V$. tejerai y $V$. vexillata), y en el segundo se consideró a todas las especies del género Vexillata en un solo trazo individual. Los cladogramas se enraizaron incluyendo un área hipotética codificada con ceros. El análisis de parsimonia se realizó con una búsqueda heurística (múltiple TBR+TBR) con los programas WinClada (Nixon 1999) y Nona (Goloboff 1993). En caso de obtenerse más de un cladograma, se calculó el consenso estricto. Con base en los cladogramas obtenidos se identificaron los clados de áreas que podrían ser interpretados como trazos generalizados (Luna-Vega \& Contreras-Medina 2000, Morrone \& Márquez 2001, Escalante et al. 2005).

\section{RESULTADOS}

Se compiló un total de 40 registros y 35 localidades únicas para el género Vexillata, así como 20,272 registros y 5,533 localidades únicas para los huéspedes (de ellas solo 18 corresponden a nuevas localidades para Vexillata y sus huéspedes, ver Cuadro 1). A partir de la matriz de datos de 25 áreas (24 provincias y un área llamada región Neártica, que comprende las localidades al norte de México) y todas las especies de Vexillata y sus huéspedes (23 en total), se obtuvieron 78 cladogramas igualmente parsimoniosos, con 45 pasos de longitud, índice de consistencia (CI) de 0.51 e índice de retención (RI) de 0.78 . El cladograma de consenso tuvo 51 pasos, $\mathrm{CI}=0.45 \mathrm{y} \mathrm{RI}$ $=0.72$ (Fig. 1). Para la matriz de 25 provincias y las 16 especies de mamíferos más el género Vexillata, se obtuvieron 112 árboles de 36 pasos, con $\mathrm{CI}=0.47$ y $\mathrm{RI}=0.80$. El cladograma de consenso estricto resultó de 46 pasos, $\mathrm{CI}=0.36$ y $\mathrm{RI}=0.70$ (Fig. 2).

En ambos cladogramas se observa una politomía basal, que incluye principalmente a las provincias de América del Sur y a la Península de Yucatán (Figs. 1 y 2). Mientras que en el cladograma derivado de la matriz que considera de manera individual 
a las especies de Vexillata, existe un clado diferenciado solo con las provincias del Páramo Norandino (NortAnPa), Costa Venezolana (VenCos) y Llanos Venezolanos (VenLlan), éstas provincias forman un clado al que se incorporan las provincias de Magdalena (Magda) y Maracaibo (Maraca) cuando se utiliza la matriz con la columna del género Vexillata. Esto se explica por la presencia de una especie de Vexillata ( $V$. tejerai) que se encuentra de manera exclusiva en las primeras tres provincias (Fig. 1; representada como el círculo negro en ese clado), información que evidentemente se pierde al utilizar el género completo. En estas provincias se encuentra también la especie de huésped Heteromys anomalus, la cual extiende su trazo individual hacia las provincias Magdalena y Maracaibo, siguiendo un patrón muy similar (representado por el círculo blanco en el mismo clado). De esta forma, $H$. anomalus se presenta como sinapomorfía en el cladograma donde se usa al género del parásito (Fig. 2; representado como el círculo negro). En este caso puede diagnosticarse un posible trazo generalizado en la costa norte de Venezuela, extendiéndose a Colombia (Fig. $3)$, justificado por estas dos especies.

El siguiente clado diferenciado en la politomía del cladograma de la Fig. 1 corresponde a las provincias Oriente de América Central (ECenAm), Occidente del Istmo de Panamá (WpanIsth), Chiapas (Chis) y Sierra Madre del Sur (SMSur), incluyendo básicamente el sur de México y América Central. Las especies que se encuentran en la base de este clado (sin ser sinapomorfías) son Heteromys desmarestianus y $H$. salvini, y aunque comparten en general el mismo patrón, éste se bifurca en la Zona de Transición Mexicana (Fig. 3). En este mismo clado se registran también las especies Heteromys irroratus, $H$. pictus y Vexillata vexillata, sin embargo, las dos primeras tienen sus límites distribucionales en el inicio de este trazo, por lo que podrían no formar parte del mismo; y la tercera, si bien su localidad más sureña es en la provincia Oriente de América Central (EcenAm), no comparte el patrón general. Cuando se utiliza todo el género Vexillata solo se observa un clado con las provincias de Chiapas y Sierra Madre del Sur (Fig. 2).

Probablemente en la provincia Magdalena se conforme un nodo biogeográfico al coincidir con el trazo generalizado de la costa norte de Venezuela (Fig. 4b). Este nodo, llamado Colombia, situado entre el trazo de la costa norte de Venezuela y el de América Central se encontraría en el noroeste de Colombia, en los límites con Panamá.

El tercer clado, sustentado por dos sinapomorfías en ambos cladogramas, corresponde al resto de México e incluye a la región Neártica. Estas sinapomorfías geográficas son dos huéspedes: Thomomys bottae y T. umbrinus (Fig. 5). No se observa un patrón evidente, sino una ramificación que incluye toda el área. Este trazo iniciaría en la región Neártica, en Estados Unidos, y termina aproximadamente a la altura de la Zona de Transición Mexicana, en el centro de México. En este lugar confluye con el trazo generalizado de América Central, generando un nodo biogeográfico en la zona 


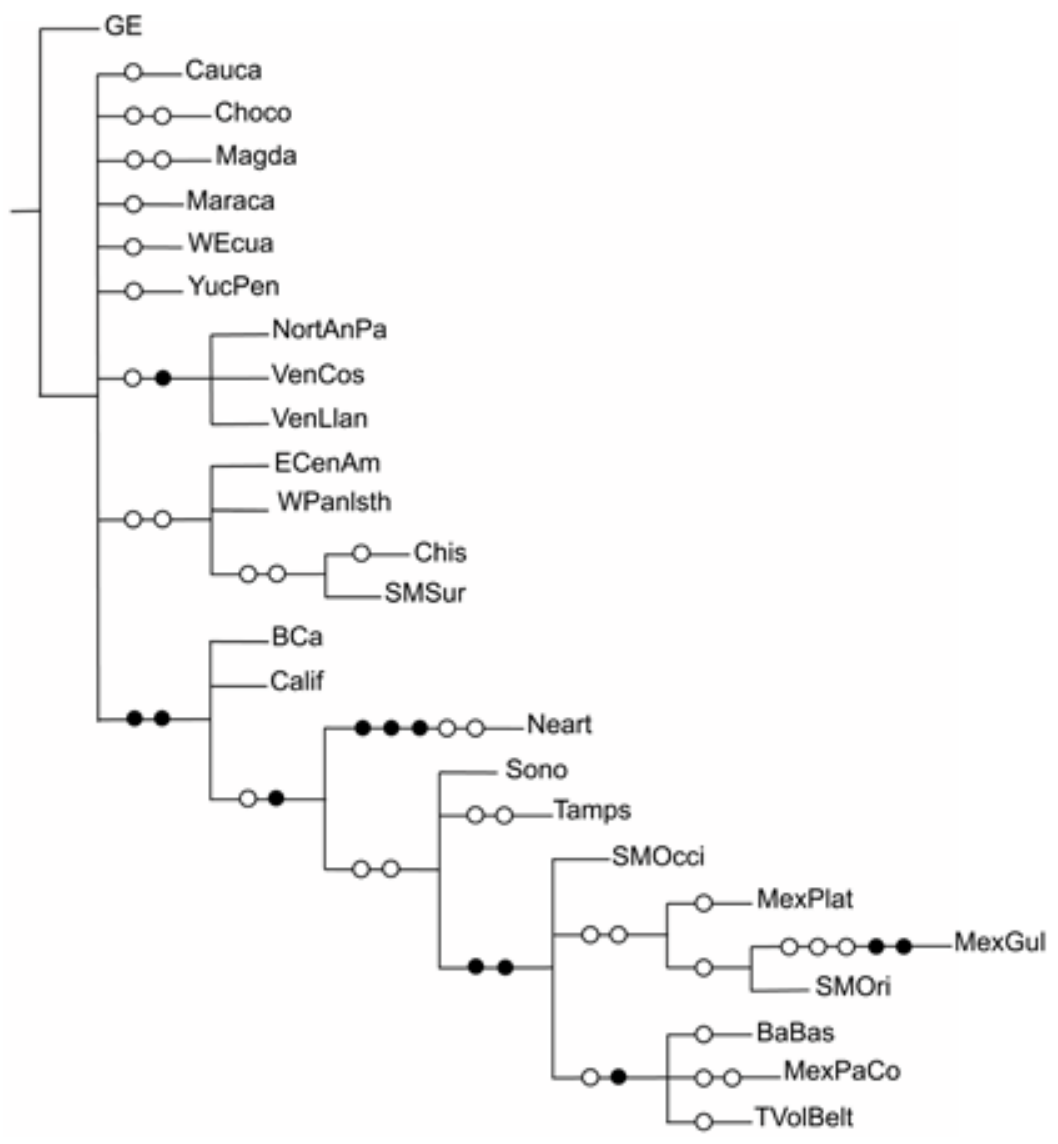

Figura 1. Cladograma de consenso estricto con 24 provincias biogeográficas, la región Neártica y 23 taxones (16 huéspedes y siete especies de Vexillata). En negro se muestran las sinapomorfías sin homoplasias, y en blanco se incluyen reversiones y convergencias. $\mathrm{GE}=$ grupo externo, $\mathrm{BaBas}=\mathrm{Cuenca}$ del Balsas, $\mathrm{BCa}=$ Baja California, Calif $=$ California; Cauca $=$ Cauca, Chis $=$ Chiapas, Choco $=$ Chocó, EcenAM $=$ Oriente de América Central, Magda $=$ Magdalena, Maraca $=$ Maracaibo, MexGul $=$ Golfo de México, $\mathrm{MexPaCo}=$ Costa Pacífica Mexicana, MexPlat $=$ Altiplano Mexicano, Neart $=$ región Neártica, NorAnPa $=$ Páramo Norandino, SMOcci $=$ Sierra Madre Occidental, SMOri $=$ Sierra Madre Oriental, SMSur $=$ Sierra Madre del Sur, Sono $=$ Sonora, Tamps $=$ Tamaulipas, TVolBelt $=$ Faja Volcánica Transmexicana, VenCos $=$ Costa venezolana, VenLlan $=$ Llanos venezolanos, YucPen $=$ Península de Yucatán, WEcua $=$ Occidente de Ecuador, WPanIsth $=$ Occidente del Istmo de Panamá. 


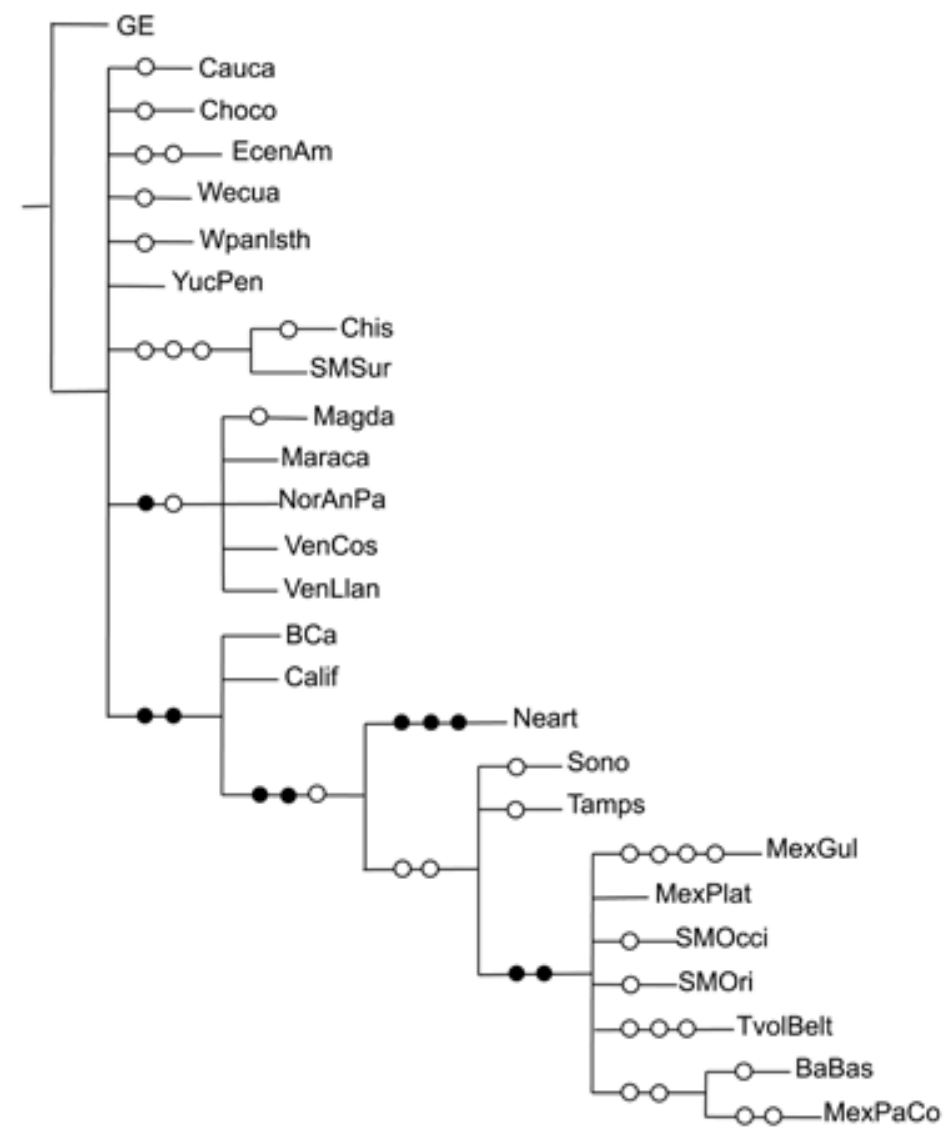

Figura 2. Cladograma de consenso estricto con 24 provincias biogeográficas, la región Neártica y 17 taxones (16 huéspedes y el género Vexillata). En negro se muestran las sinapomorfías sin homoplasias, y en blanco se incluyen reversiones y convergencias. Para los acrónimos de las provincias, figura 1.

central de México, llamado Centro de México (Fig. 4a). Otras dos especies, Chaetodipus hispidus y Perognathus flavus (la primera solo en la matriz con Vexillata como género) comparten un patrón similar, excepto porque no se las encuentra en la Península de Baja California (BCa y Calif). Más adelante, y excluyendo a las provincias de Sonora (Sono) y Tamaulipas (Tamps) y la región Neártica (Neart), existe un clado sustentado por dos especies de mamíferos, Cratogeomys merriami y C. tylorhinus, ambas de la zona central de México. Estas especies diagnosticarían un trazo anidado, incluyendo las provincias de la Zona de Transición Mexicana (Sierra Madre Occiden- 


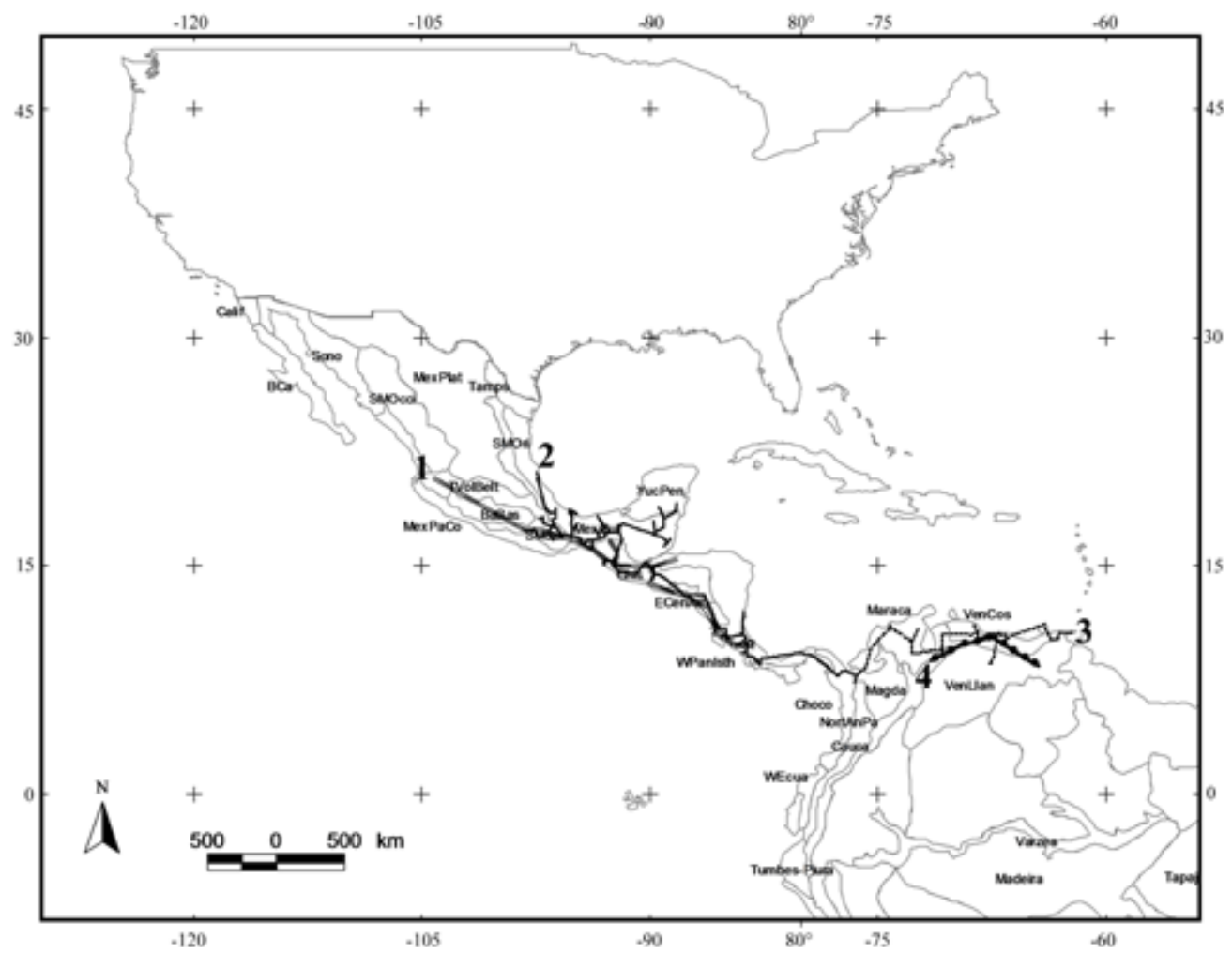

Figura 3. Trazos individuales de (1) Heteromys salvini y (2) H. desmarestianus en México y América Central; y (3) Heteromys anomalus y (4) Vexillata tejerai en el norte de América del Sur.

tal, Sierra Madre Oriental, Cuenca del Balsas y Faja Volcánica Transmexicana), así como al Altiplano Mexicano, y las provincias del Golfo y el Pacífico. Finalmente, se observan algunas especies de Vexillata ( $V$. convoluta, $V$. liomyos y $V$. legalle) que se encuentran de manera exclusiva en este trazo (Fig. 1).

\section{DISCUSIÓN}

El conocimiento de la distribución geográfica de los parásitos, en ocasiones, parece estar limitado a la proporción del esfuerzo realizado por los especialistas (Guerrero, 1985). A partir de la base de datos construida, es posible observar que la cantidad de datos sobre la distribución geográfica de los parásitos y sus huéspedes no es equiparable, ya que el número de registros analizados para los huéspedes es de 20,272, mientras que para los parásitos solo se cuenta con 40 registros, lo cual equivale al $0.19 \%$ de los datos conocidos para la distribución de los huéspedes de Vexillata. Hasta el momento, ésta es la única información con la que se cuenta, y dado que existen muy 


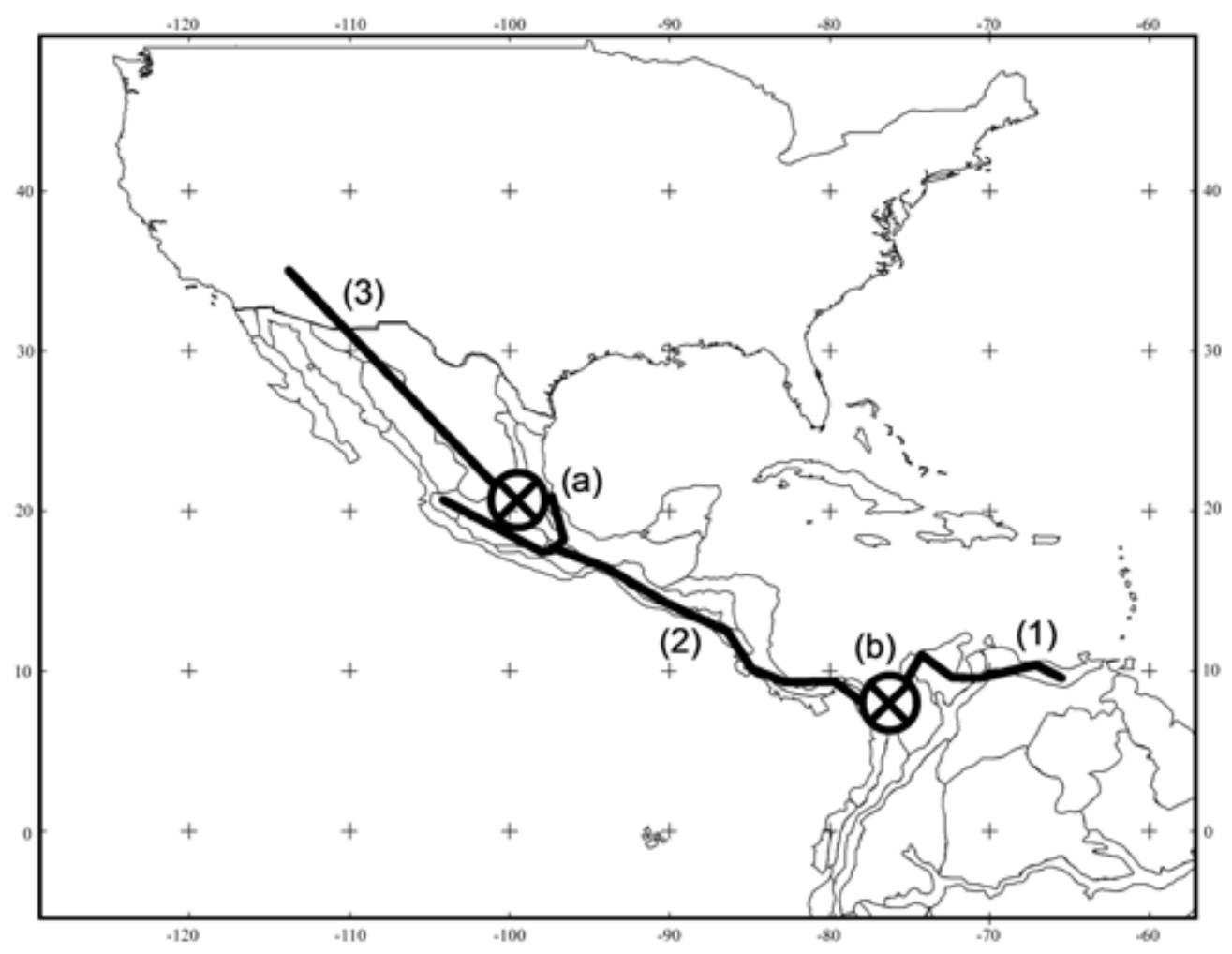

Figura 4. Trazos generalizados de (1) la costa norte de Venezuela, (2) América Central y (3) Neártico; y posibles nodos panbiogeográficos en (a) el Centro de México y (b) Colombia.

pocos datos sobre la distribución para este grupo de parásitos, resulta indispensable adicionar información a partir de nuevas recolectas, en localidades inexploradas para los parásitos, principalmente a partir de aquellos con los que se cuenta con los registros de la distribución de los huéspedes, y así corroborar y contrastar los patrones mostrados en el presente trabajo.

La mayoría de los análisis panbiogeográficos han sido realizados para un solo grupo de estudio (por ejemplo, helmintos de peces, aves, insectos, mamíferos, plantas), en ocasiones cercanamente relacionados filogenéticamente. Sin embargo, en este trabajo abordamos el estudio de dos taxones no relacionados filogenéticamente, pero con una relación ecológica obligatoria (para el parásito) que completa su ciclo de vida en el mamífero. En la literatura se ha recomendado que los análisis panbiogeográficos se realicen con taxones lejanos filogenéticamente, evitando analizar un solo taxón, lo cual daría mayor robustez a los análisis (Croizat 1958, Contreras-Medina et al. 2001). En ambos sentidos, nuestro artículo cumple con dos propósitos: uno, realizar un aná- 

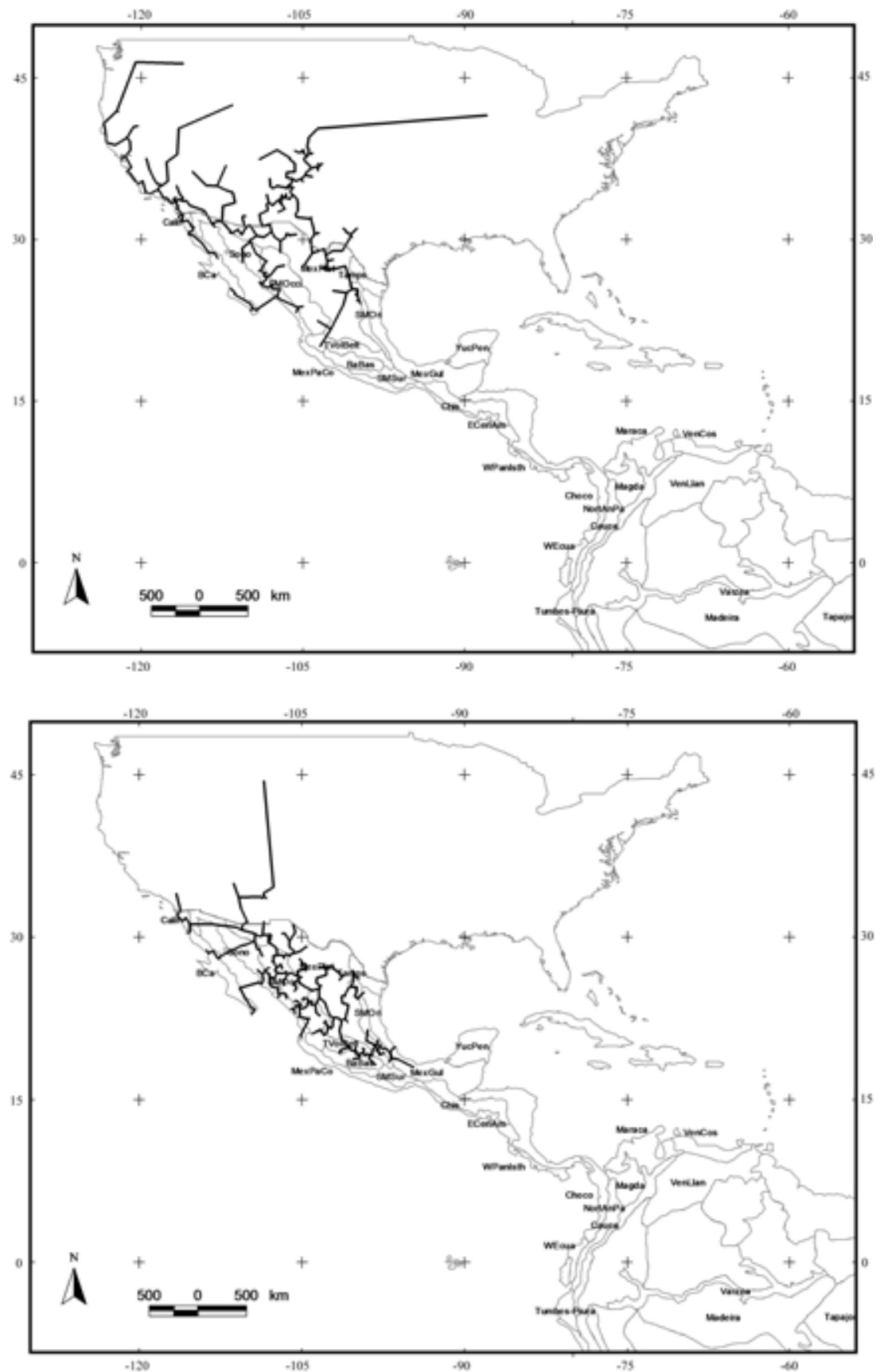

Figura 5. Trazos individuales de (a) Thomomys bottae y (b) T. umbrinus. 
lisis integrador con taxones no relacionados filogenéticamente; y dos, estudiar los patrones de distribución de dos taxones ecológicamente relacionados, en donde podría haber existido una historia de coevolución por colonización y/o descendencia.

En nuestros resultados no encontramos evidencia clara de patrones compartidos y exclusivos entre parásitos y huéspedes. En ninguno de los clados se observó la presencia de sinapomorfías de mamíferos y helmintos parásitos, excepto en el caso donde Heteromys anomalus y Vexillata tejerai comparten un clado en la matriz con las especies de parásitos. Solo se tiene un registro de $V$. tejerai publicado por Guerrero (1984) y en el presente trabajo se adicionan cinco nuevas localidades en la distribución geográfica de este nemátodo, el cual se restringe hasta el momento a Venezuela. Por otro lado, en Venezuela también existe un solo registro de $V$. scorzai para este mismo huésped (Guerrero 1984), por lo que podemos considerar a estas especies de parásitos como endémicas a América del Sur.

Si existiera más información sobre la distribución geográfica de $V$. scorzai podría corroborarse el trazo que incluye a esta especie. Incluso en la matriz donde las especies de Vexillata son tratadas como un género, éste se presenta como homoplasia dispersa en los diferentes clados y con índices muy bajos $(\mathrm{ci}=0.10, \mathrm{ri}=0.10)$. En este caso, los mamíferos presentan patrones biogeográficos más definidos, debido a que se cuenta con mayor información sobre su distribución geográfica en comparación con la de los parásitos, como ya se mencionó.

Hasta el momento Heteromys desmarestianus es el único huésped con una distribución amplia, en cual aloja más de una especie de Vexillata $(V$. brooksi, $V$. dessetae, $V$. legalle y Vexillata sp.), pero solo $V$. dessetae y $V$. legalle se han registrado en simpatría en la región de los Tuxtlas, Veracruz. De estas especies, solo $V$. vexillata está ampliamente distribuida (desde Norteamérica hasta localidades de Costa Rica), en comparación con $V$. brooksi, $V$. dessetae y $V$. legalle, las cuales presentan distribuciones hasta el momento como restringidas en las regiones Neotropicales (México y Costa Rica).

Se puede observar este sesgo en el escaso conocimiento sobre la helmintofauna y distribución de los endoparásitos en huéspedes como Heteromys anomalus en América del Sur o Thomomys bottae para América del Norte, o bien de aquellos mamíferos aún no examinados como los del género Dipodomys en México. Por otra parte, algunos de los grupos de mamíferos no explorados hasta el momento se encuentran en peligro de extinción (NOM-059-ECOL-200, Semarnat 2002) y con ellos, la biodiversidad de sus helmintos parásitos. Por esta razón es importante contar con distintos estudios, tales como los análisis de distribución potencial o biogeográficos cladísticos, que nos permitan conocer más sobre sus patrones biogeográficos (v. gr. Escalante et al. 2007).

Al comparar nuestros resultados con trazos y nodos de otros taxones, podemos observar que ninguno de los nodos encontrados en otros trabajos con helmintos pará- 
sitos coincide con la información aquí presentada (Rosas-Valdez \& Pérez-Ponce de León 2005, 2008, Martínez-Aquino et al. 2007). Sin embargo, es difícil establecer una comparación con otros estudios dado el nivel de universalidad del nuestro. Aún así, hemos podido observar que el nodo Centro de México coincide con el hecho de que se han encontrado nodos biogeográficos para otros taxones en esta parte del país: aves (Álvarez-Mondragón \& Morrone 2004), mamíferos (Escalante et al. 2004, García-Marmolejo et al. 2008), pulgas (Morrone y Gutiérrez 2005) y coleópteros (Márquez \& Morrone 2003, Corona et al. 2009), entre otros. En el caso de América Central y del Sur, varios trazos generalizados identificados por Quijano-Abril et al. (2006; trazos 2, 15, 14 y 21) para un género de plantas, coinciden con nuestros trazos, así como un trazo generalizado y un nodo identificado para otro género de plantas (Franco \& Berg 1997) coinciden espacialmente con nuestro nodo Colombia. También los trazos generalizados y el nodo de América ("gate") originalmente propuestos por Croizat (1958) coinciden con lo encontrado en este estudio.

Poco se conoce sobre la historia biogeográfica del grupo. Durette-Desset (1971), en su trabajo sobre los heligmosómidos, dividió al género Vexillata en dos grupos, A y $\mathrm{B}$, presentando dos propuestas sobre el posible origen del género. La primera plantea que el grupo denominado A (especies que se distribuyen en América del Norte y Central) se originó directamente de los Heligmonellinae, mientras que el grupo B (especies que se localizan en América del Sur) tiene un origen a partir de Travassostrongylus, parásito exclusivo de marsupiales sudamericanos. La segunda propuesta establece el origen del grupo B a partir del grupo A con base en la distribución geográfica de sus huéspedes. Con nuestros resultados, podríamos proponer que existen más bien tres componentes bióticos: de América del Norte, de América Central y de América del Sur, los cuales han evolucionado de manera independiente, pero que han entrado en contacto en los sitios identificados como nodos. Franco (2001), siguiendo a Croizat (1976), sugiere para Cecropia, que la zona del trazo generalizado de América Central hasta Colombia es parte de una gran masa terrestre antigua. Pero por otro lado, dado que nuestros taxones dependen de los mamíferos para completar sus ciclos de vida, quizá sus patrones de distribución estén más influidos por la formación del Istmo de Panamá y su dispersión a través de él (Simpson 1940, 1950, Marshall et al. 1982).

La historia evolutiva compartida de los huéspedes y sus parásitos es multidimensional, si esta asociación es producto de una coevolución por descendencia (sus ancestros se asociaron entre sí en el pasado y las especies heredaron dicha asociación) o por colonización (una de las especies se originó en otro huésped y subsecuentemente se relacionaron por transferencia o dispersión), o si dentro de la historia hay más de un proceso involucrado (Brooks \& McLennan 1993, Morrone, 2004), entonces podríamos conocer más acerca de su diversificación.

Las explicaciones biogeográficas en otros sistemas de nemátodos parásitos de mamíferos en América son escasas, complejas y poco exploradas. Algunas expli- 
caciones recurrentes de los patrones biogeográficos encontrados en estos trabajos, están relacionadas con procesos de dispersión y posterior especiación secundaria en ciertas localidades que ha dado origen a distribuciones restringidas (probablemente correspondientes a etapas diferenciales de movilidad e inmovilidad de los taxones, de acuerdo con Croizat). Estos procesos se han observado en algunas especies del género Viannaia ( $V$. cayennensis y $V$. barusi), parasitos intestinales de Didelphis (Guerrero, 1985). Por otro lado se han propuesto eventos vicariantes de especiación en Trypanoxyuris, parásito intestinal de Callicebus (Hugot et al., 1994); o aislamiento entre las poblaciones asociadas a refugios del Cuaternario, para el caso de Monodelphoxyuris dollmeiri, parásito del colon de Monodelphis emiliae (Guerrero \& Hugot, 2003).

El estudio de los parásitos adicionalmente nos provee indirectamente de información de la historia biogeográfica de sus huéspedes, y a su vez, la historia de los huéspedes permite dilucidar los eventos de especiación en los parásitos (Guerrero, 1985; Hugot et al., 1994; Guerrero \& Hugot, 2003). Tradicionalmente, se conoce que Heteromys fue uno de los últimos grupos norteamericanos que se dispersaron hacia América del Sur (Hershkovitz 1972, Simpson 1980), el tiempo, así como el número de eventos de colonización siguen siendo controversiales (Anderson \& Soriano 1999, Anderson et al. 2002, Anderson \& Jarrin 2002). Actualmente, Heteromyidae tiene una distribución amplia, mientras que su grupo hermano, los Geomyidae, que también se dispersaron de América del Norte hacia el Sur, poseen una distribución en el sur sólo al extremo oeste-noroeste (Handley 1976, Anderson 1999). Estudios recientes que incluyen filogenias (a nivel de especies) y modelado de la distribución geográfica, entre otras investigaciones, han demostrado que el ingreso de los Heteromys a América del Sur sigue siendo una controversia, dado que la historia biogeográfica de este grupo de huéspedes es compleja (Anderson 1999, Anderson \& Soriano 1999, Anderson et al. 2002, Anderson \& Jarrin 2002).

A pesar de este hecho y con estos antecedentes, el nodo y trazos en Centroamérica en el caso de los Geomyoidea, aportan información valiosa al considerar que dentro de este sistema de parásitos-mamíferos, ocurrió una posible especiación por descendencia, por ejemplo, el intercambio biótico de los parásitos entre un grupo de mamíferos co-distribuidos dado los eventos de dispersión de sus huéspedes. Por este hecho, podríamos considerar también, en el caso de los Heteromys en América del Sur, que en este grupo también ocurrió un evento de especiación y subsiguiente dispersión, por lo que algunas poblaciones quedaron aisladas ( $V$. scorzai), mientras que otras pueden tener una distribución amplia ( $V$. tejerai), que lograron co-evolucionar y extender su área de distribución paralelamente con su huésped, como es el caso de Heteromys anomalus y las especies de Vexillata mencionadas.

En panbiogeografía los nodos son interpretados como zonas de convergencia tectónica y biótica (Morrone, 2004), los cuales han sido empleados como evidencia para proponer áreas para conservación (Álvarez-Mondragón \& Morrone 2004, Martínez- 
Aquino et al. 2007). En el presente trabajo, logramos detectar dos posibles nodos (Colombia y Centro de México, Fig. 4), y dado que solo el nodo sudamericano provee información sobre la convergencia biótica entre Vexillata y sus huéspedes, éste podría representar un área de importancia para la conservación. Sin embargo, será necesario realizar análisis de distribución con estos datos mediante modelos predictivos, que nos permitan obtener mayor información. El nodo Centro de México se encuentra ubicado en una zona donde ya se han realizado algunos estudios para establecer prioridades de conservación de los mamíferos (Fuller et al. 2006), donde de manera indirecta, también se propone conservar su helmintofauna.

La posible historia sobre la diversificación del género Vexillata es compleja (ej. $V$. vexillata y $V$. convoluta parasitan, cada uno, cinco huéspedes de dos géneros), y será necesario contrastar los patrones obtenidos en el presente trabajo con estudios biogeográficos de homología secundaria (biogeografía cladística), basados en estudios filogenéticos sólidos de los grupos de huéspedes del género Vexillata y de otros taxones que habitan en las mismas áreas.

AGRADECIMIENTOS. Los autores agradecen a Jesús A. Fernández, por la ayuda brindada en los datos de las distribuciones geográficas de algunas especies analizadas, y a David J. Hafner por la recolecta y envío de Thomomys bottae de Nuevo México. Patricia Illoldi, Juan J. Morrone y Gerardo Rodríguez leyeron críticamente el manuscrito preliminar. T. Escalante agradece el apoyo del programa PROFIP-DGAPA. J. Falcón-Ordaz agradece el apoyo del programa "Estancias Posdoctorales y Sabáticas Vinculadas al Fortalecimiento de la Calidad del Posgrado Nacional, 2008” de CONACyT. E. A. Martínez-Salazar agradece a CONACyT por la beca posdoctoral, en el programa "Estancias posdoctorales y sabáticas al extranjero para la consolidación de grupos de Investigación”. Parte de esta investigación se realizó con el apoyo del proyecto 80370 de CONACyT.

Las colecciones consultadas incluyen a: Burke Museum; California Academy of Sciences; CNMA/ Coleccion Nacional de Mamiferos; Colección de Mamíferos de la Sierra Volcánica Transversal de México (UAM-I); Colección de Mamíferos de Nuevo León, México (UANL); Cornell University Museum of Vertebrates; Field Museum; Florida Museum of Natural History; GBIF-Sweden; Los Angeles County; Museum of Natural Science, Louisiana State University Museum of Natural Science; LSUMZ; Marine Science Institute; UCSB; Michigan State University Museum; MSB Mammals Specimens; Museum of Comparative Zoology, Harvard University; Museum of Texas Tech University; Museum of Vertebrate Zoology; NatureServe Network Species Occurrence Data; Paleobiology Database; Royal Ontario Museum; Sternberg Museum of Natural History; UCD Database Schema for UC Davis Wildlife Museum; University of Alaska Museum of the North; University of Kansas Biodiversity Research Center; University of Minnesota Bell Museum of Natural History; University of Nebraska State Museum; University of Tennessee - Chattanooga (UTC); University of Texas at El Paso; UNSM Vertebrate Specimens; Utah Museum of Natural History; WNMU Mammals Specimens; y Yale University Peabody Museum.

\section{LITERATURA CITADA}

Aguilar-Aguilar, R., R. Contreras-Medina \& G. Salgado-Maldonado. 2003. Parsimony Analysis of Endemicity (PAE) of Mexican Hydrological based on helminth parasites of freshwater fishes. Journal of Biogeography, 30: 1861-1872. 
Aguilar-Aguilar, R., G. Salgado-Maldonado, R. Contreras-Medina \& A. Martínez-Aquino. 2008. Richness and endemism of helminth parasites of freshwater fishes in Mexico. Biological Journal of the Linnean Society, 94: 435-444.

Álvarez-Mondragón, E. \& J. J. Morrone. 2004. Propuesta de áreas para la conservación de aves de México, empleando herramientas panbiogeográficas e índices de complementariedad. Interciencia, 29: 112-120.

Anderson, R. P. 1999. Preliminary review of the systematics and biogeography of the spiny pocket mice (Heteromys) of Colombia. Revista de la Academia Colombiana de Ciencias. Exactas, Físicas y Naturales, 23(suplemento especial): 613-630.

Anderson, R. P., M. Gómez-Laverde \& A. T. Peterson. 2002. Geographical distributions of spiny pocket mice in South America: insights from predictive models. Global Ecology and Biogeography, 11: 131-141.

Anderson, R. P. \& P. Jarrin V. 2002. A new species of spiny pocket mouse (Heteromyidae: Heteromys) Endemic to Western Ecuador. American Museum Novitates, 3382: 1-26.

Anderson, R. P. \& P. J. Soriano. 1999. The occurrence and biogeographic significance of the southern spiny pocket mouse Heteromys australis in Venezuela. Zeitschrift für Säugetierkunde, 64: 121-125.

Brooks, D. R. \& D. McLennan. 1993. Parascript: Parasites and the language of evolution. Smithsonian Institution Press. Washington, D.C.

Brooks, D. R., V. León-Règagnon \& G. Pérez-Ponce de León. 2001. Los parásitos y la biodiversidad. Pp. 245-289. In: H. M. Hernández, A. N. García A., F. Alvarez y M. Ulloa (eds.) Enfoques contemporáneos para el estudio de la biodiversidad. Instituto de Biología, UNAM-Fondo de Cultura Económica, México, D. F.

Caballero, E. 1958. Estudios helmintológicos de la región oncocercosa de México y de la República de Guatemala. Nematoda: 10a parte. Anales de la Escuela Nacional de Ciencias Biológicas, 9: 64-66.

Caballero, E. \& M. C. Cerecero. 1943. Longistriata convoluta n. sp. (Nematoda: Trichostrongylidae) parásito del intestino de una "tuza" Cratogeomys merriami (Thomas). Anales de la Escuela Nacional de Ciencias Biológicas, 14: 201-205.

Contreras-Medina, R., I. Luna-Vega \& J. J. Morrone. 2001. Conceptos biogeográficos. Elementos, 41(8): 33-37.

Corona, A. M., V. H. Toledo \& J. J. Morrone. 2009. Track analysis of the Mexican species of Buprestidae (Coleoptera): testing the complex nature of the Mexican Transition Zone. Journal of Biogeography, 36: 1730-1738.

Crisci, J. V., L. Katinas \& P. Posadas. 2000. Introducción a la teoría y práctica de la biogeografía histórica. Sociedad Argentina de Botánica. Buenos Aires.

Croizat, L. 1958. Panbiogeography: Vols. 1 y 2. Publicado por el autor, Caracas.

Croizat, L. 1964. Space, time, form: The biological synthesis. Publicado por el autor. Caracas.

Croizat, L. 1976. Biogeografía analítica y sintética (Panbiogeografia) de las Américas. Vols. I y II. Biblioteca de la Academia de Ciencias Físicas, Matemáticas y Naturales 16, Caracas.

Denke, A. M. 1977. Quatre nouveux nématodes héligmosomes parasites de rongeurs du Mexique. Bulletin du Muséum National d'Histoire Naturelle, 327: 777-787.

Durette-Desset, M. C. 1970. Description de Vexillata petteri n. sp. nématode héligmosome parasite d'un rongeur néarctique. Annales de Parasitologie Humaine et Comparé, 45: 289-293.

Durette-Desset, M. C. 1971. Essai de classification des nématodes héligmosomes. Corrélations avec la paléobiogéographie des hôstes. Mémories du Muséum National d'Histoire Naturelle, 69: 126 p. 
Durette-Desset, M. C. 1978. Nouvelles données morphologiques sur des nématodes Trichostrongyloides des collections du United States National Museum. Bulletin du Muséum National d'Histoire Naturelle, 352: 135-147.

Durette-Desset, M. C. \& M. C. Digiani. 2005. Systematic position of some neartic Heligmosomoidea (Nematoda: Trichostrongylina) from the U.S. National Parasite collection and their description. Journal of Parasitology, 91: 893-899.

Escalante, T., Estrada, P. Illoldi, P., Linaje, M., Neira F., Rivas, M. Rodríguez, G. \& J. Solervicens. 2007. Patrones de distribución geográfica de Eurymetopum (Coleoptera, Cleridae) con base en modelos de nicho ecológico. Darwiniana, 45(suplemento): 55-131.

Escalante, T., G. Rodríguez \& J. J. Morrone. 2004. The diversification of Nearctic mammals in the Mexican Transition Zone. Biological Journal of the Linnean Society, 83: 327-339.

Escalante, T., Rodríguez, G. \& J. J. Morrone. 2005. Las provincias biogeográficas del Componente Mexicano de Montaña desde la perspectiva de los mamíferos continentales. Revista Mexicana de Biodiversidad, 76: 199-205.

ESRI. 1999. ArcView 3.2 GIS Environmental Systems Research Institute. Nueva York.

Falcón-Ordaz J., \& García-Prieto L. 2005. New species of Vexillata (Nematoda: Trichostrongylina: Ornithostrongylidae) parasite of Heteromys desmarestianus (Rodentia: Heteromyidae) from Costa Rica. Journal of Parasitology, 91: 329-334.

Falcón-Ordaz, J., S. L. Gardner, \& G. Pérez-Ponce De León. 2001. Vexillata liomyos n. sp. (Nemata: Ornithostrongylidae) from Liomys pictus (Rodentia: Heteromyidae) from Mexico, with comments on the synlophe of Vexillata armandae. Journal of Parasitology, 87: 656-659.

Falcón-Ordaz J., Hsuan-Wien Chen, \& R. Lamothe A. 2006. A new species of Vexillata (Nematoda: Ornithostrongylidae) parasite of attwater's pocket gopher from Texas. Journal of Parasitollogy, 92: 595-599.

Falcón-Ordaz J. \& M. A. Sanabria-Espinosa. 1997. Vexillata vexillata (Nematoda: Ornithostrongylidae) parásito de roedores de México. Revista de Biología Tropical, 45: 1691-1694.

Franco, P. 2001. Estudios panbiogeográficos en Colombia. Pp. 221-224. In: J. Llorente-Bousquets \& J. J. Morrone (eds.). Introducción a la biogeografía en Latinoamérica: teorías, conceptos, métodos y aplicaciones. Las Prensas de Ciencias, UNAM, México, D. F.

Franco, P. \& C. C. Berg. 1997. Distributional patterns of Cecropia (Cecropiaceae) a panbiogeographic analysis. Caldasia, 19(1-2): 285-296.

Fuller, T., M. Munguía, M. Mayfield, V. Sánchez-Cordero \& S. Sarkar. 2006. Incorporating connectivity into conservation planning: A multi-criteria case study from Central Mexico. Biological Conservation, 133: 131-142.

García-Marmolejo, G., T. Escalante \& J. J. Morrone. 2008. Establecimiento de prioridades para la conservación de mamíferos terrestres neotropicales de México. Mastozoología Neotropical, 15(1): 41-65.

Gardner, S. L., E. B. Fong, L. Al-Banna, \& S. T. Raymond. 1994. A new species of Vexillata (Nemata: Ornithostrongylidae) from the coarse-haired pocket mouse Chaetodipus hispidus in New Mexico. Journal of Parasitology, 80: 591-594.

Goloboff, P. 1993. Nona ver. 2. Publicado por el autor, San Miguel de Tucumán.

Guerrero, R. 1984. Nematoda: Trichostrongyloidea parasites of Venezuelan wild mammals III. The genus Vexillata Travassos, 1937. Annales de Parasitologie Humaine et Comparée, 59: 253-261.

Guerrero, R. 1985. Nematoda: Trichostrongyloidea Parásitos de mamíferos silvestres de Venezuela. II Revisión del género Viannia Travassos, 1914. Memoria de la Sociedad de Ciencias Naturales La Salle, XLV(124): 9-47. 
Guerrero, R. \& J. P. Hugot. 2003. Monodelphoxyuris dollmeiri n. gen., n. sp. (Nematoda. Oxyuridae) from Monodelphis emiliae (Marsupialia: Didelphidae) in Perú. Systematic Parasitology, 55: 73-79.

Hall, M. C. 1916. Nematodes parasites of the orders Rodentia, Lagomorpha and Hyracoidea. Proceedings of the United States National Museum, 50: 123-160.

Hall, E. R. 1981. The mammals of North America. 2nda. ed. John Wiley and Sons, Nueva York.

Handley, C. O., Jr. 1976. Mammals of the Smithsonian Venezuelan Project. Brigham Young University Science Bulletin, Biological Series, 20(5): 1-91.

Hershkovitz, P. 1972. The recent mammals of the Neotropical Region: A zoogeographic and ecological review. Pp. 311-431. In: Keast, A., F. C. Erk \& B. Glass (eds.), Evolution, mammals, and southern continents, State University of New York Press, Albany.

Hoberg, E. P. 1997. Phylogeny and historical reconstruction: Host-parasite systems as keystones in biogeography and ecology. Pp. 243-261. In: M. L. Reaka-Kudla, D. E. Wilson y E. O. Wilson (eds.). Biodiversity II: Understanding and protecting our biological resources, Joseph Henry Press, Washington D. C.

Hugot, J. P., S. Morand \& R. Guerrero. 1994. Trypanoxyuris croizati n.sp. and T. callicebi Hugot \& Vaucher,1985 (Nematoda: Oxyuridae), two vicariant form parasitic in Callicebus spp. (Primatia, Cebidae). Systematic Parasitology, 27: 35-43.

Luna Vega I., O. Alcántara A., J. J. Morrone, \& D. Espinosa O. 2000. Track analysis and conservation priorities in the cloud forests of Hidalgo, Mexico. Diversity and Distributions, 6: 137-143.

Luna-Vega, I. \& R. Contreras-Medina. 2000. Distribution of the genera of Theaceae (Angiospermae: Theales): A panbiogeographic analysis. Biogeographica, 76: 79-88.

Márquez, J. \& J. J. Morrone. 2003. Análisis panbiogeográfico de las especies de Homalolinus y Heterolinus (Coleoptera: Staphylinidae: Xantholinini). Acta Zoológica Mexicana (nueva serie), 90 : $15-25$.

Marshall L. G., S. D.Webb, J. J. Sepkoski \& D. M. Raup. 1982. Mammalian evolution and the Great American Interchange. Science, 215: 1351-1357.

Martínez-Aquino, A., R. Aguilar-Aguilar, H. O. Santa Anna del Conde-Juárez \& R. ContrerasMedina. 2007. Empleo de herramientas panbiogeográficas para detectar áreas para conservar: Un ejemplo con taxones dulceacuícolas. Pp. 449-460. In: Luna,I., J. J. Morrone \& D. Espinosa (Eds). Biodiversidad de la Faja Volcánica Transmexicana. Las Prensas de Ciencias, UNAM, México, D. F.

Morrone, J. J. 1994. On the identification of areas of endemism. Systematic Biology, 43: 438-441.

Morrone, J. J. 2001 Biogeografía de América Latina y El Caribe. M \& T-Manuales \& Tesis SEA, Vol. 3, Sociedad Entomológica Aragonesa, Zaragoza.

Morrone, J. J. 2004. Panbiogeografía, componentes bióticos y zonas de transición. Revista Brasileira de Entomologia, 48: 149-162.

Morrone, J. J. 2006. Biogeographic areas and transition zones of Latin America and the Caribbean Islands based on panbiogeographic and cladistic analyses of the Entomofauna. Annual Review of Entomology, 51: 467-494.

Morrone, J. J. \& A. Gutiérrez. 2005. Do fleas (Insecta: Siphonaptera) parallel their mammal host diversification in the Mexican transition zone? Journal of Biogeography, 32: 1315-1325.

Morrone, J. J. \& J. Márquez. 2001. Halffter's Mexican Transition Zone, beetle generalized tracks, and geographical homology. Journal of Biogeography, 28: 365-650.

Nixon, K.C. 1999. Winclada (beta) ver. 0.9.9. Publicado por el autor, Ithaca, NuevaYork.

Pérez-Ponce de León, G. 2003. Biodiversity and biogeographic patterns in the Mesa Central of México: Insights from host-parasite systems. Journal of Parasitology, 89(Suppl.): S126-S133. 
Pérez-Ponce de León, G. \& A. Choudhury. 2002. Adult endohelminth parasites of ictalurid fishes (Osteichthyes: Ictaluridae) in Mexico: Empirical evidence for biogeographical patterns. Comparative Parasitology, 69: 10-19.

Pérez-Ponce de León, G. \& A. Choudhury. 2005. Biogeography of helminth parasites of freshwater fishes in Mexico: The search for patterns and processes. Journal of Biogeography, 32: 645-659.

Quijano-Abril M. A., R. Callejas-Posada \& D. R. Miranda-Esquivel. 2006. Areas of endemism and distribution patterns for Neotropical Piper species (Piperaceae). Journal of Biogeography, 33(7): 1266-1278.

Rojas-Parra, C. A. 2007. Una herramienta automatizada para realizar análisis panbiogeográficos. Biogeografia, 1: 31-33.

Rosas-Valdez, R. \& G. Pérez-Ponce de León. 2005. Biogeografía histórica de helmintos parásitos de ictalúridos en América del Norte: Una hipótesis preliminar utilizando el método panbiogeográfico. Pp. 217-226. In: J. Llorente B. y J. J. Morrone (Eds). Regionalización biogeográfica en Iberoamérica y tópicos afines: Primeras Jornadas Biogeográficas de la Red Iberoamericana de Biogeografia y Entomología Sistemática (RIBES XII.I CYTED). Las Prensas de Ciencias, Facultad de Ciencias, UNAM, México, D. F.

Rosas-Valdez, R. \& G. Pérez-Ponce de León. 2008. Composición taxonómica de los helmintos parásitos de ictalúridos y heptaptéridos (Osteichthyes: Siluriformes) de México, con una hipótesis de homología biogeográfica primaria. Revista Mexicana de Biodiversidad, 79: 473- 499.

Rosas-Valdez, R., O. Domíguez-Domíguez, A. Choudhury \& Gerardo Pérez-Ponce de León. 2007. Helminth parasites of the Balsas catfish Ictalurus balsanus (Siluriformes: Ictaluridae) in several localities of the Balsas River drainage, Mexico: Species composition and biogeographical affinities. Comparative Parasitology, 74: 204-210.

Schmidly, D. J., K. T. Wilkins \& J. M. Der. 1993. Biogeography: Biology of the Heteromyidae. Pp. 319-356. In: Genoways, H. H. \& J. H. Brown Special (Eds.) Publication No. 10, The American Society of Mammalogists.

Semarnat. 2002. Norma Oficial Mexicana NOM-Ecol-059-2001. Especies nativas de México de flora y fauna Silvestres. Categorías de Riesgo y especificaciones para su inclusión, exclusión o cambio - Lista de especies en riesgo. Diario Oficial de la Federación, Segunda sección de Medio Ambiente y Recursos Naturales. 6 de marzo de 2002. México, D. F.

Simpson, G. G. 1940. Mammals and land bridges. Journal of the Washington Academy of Sciences, 30: 137-163 312 .

Simpson, G. G. 1950. History of the fauna of Latin America. American Scientist 38: 361-389.

Simpson, G. G. 1980. Splendid isolation: the curious history of South American mammals. Yale University Press, New Haven.

Vidal-Martínez, V. M. \& C. R. Kennedy. 2000. Zoogeographical determinants of the composition of the helminth fauna of Neotropical cichlid fish. Pp. 227- 290. In: Salgado-Maldonado, G., A. N. García-Aldrete y V. M. Vidal-Martínez (eds.), Metazoan parasites in the Neotropics: A systematic and ecological perspective, Instituto de Biología, UNAM, México, D. F.

Villa, R. B. \& F. A. Cervantes. 2003. Los mamíferos de México. Grupo Editorial Iberoamericana, S. A. de C. V. México, D. F.

Wilson, D. E. \& D. A. M. Reeder. 2005. Mammal species of the world: A taxonomic and geographic reference, 3a ed. Johns Hopkins University Press, Baltimore.

Yoyotte, V. E. 1972. Étude de huit nématodes parasites de vertébrés du Venezuela et de Colombie. Bulletin du Muséum National d'Histoire Naturelle, 35: 477-498. 


\section{APÉNDICE}

Número de localidades únicas registradas para las especies huéspedes y parásitas.

Huéspedes:

Chaetodipus hispidus 428

Cratogeomys fulvescens 7

Cratogeomys merriami 128

Cratogeomys thylorinus 97

Geomys attwateri 35

Geomys pinetis 36

Heteromys anomalus 52

Heteromys australis 9

Heteromys desmarestianus 277

Heteromys irroratus 1685

Heteromys pictus 1869

Heteromys salvini 79

Perognathus flavus 693

Thomomys bottae 483

Thomomys talpoides 697

Thomomys umbrinus 521

Parásitos:

Vexillata armandae 3

Vexillata brooksi 1

Vexillata chabaudi 1

Vexillata chitwoodi 1

Vexillata convoluta 7 (1 sin georreferencia precisa)

Vexillata dessetae 2

Vexillata geomyos 1

Vexillata legalle 2

Vexillata liomyos 4

Vexillata noviberae 0 ( $\sin$ georreferencia precisa)

Vexillata scorzai 1

Vexillata tejerai 6

Vexillata vexillata 6

Vexillata sp. 2 